\title{
Self-Scaled Barrier Functions on Symmetric Cones and Their Classification
}

\author{
Raphael A. Hauser ${ }^{1}$ and Osman Güler ${ }^{2}$ \\ ${ }^{1}$ Department of Applied Mathematics and Theoretical Physics (DAMTP) \\ University of Cambridge \\ Silver Street \\ Cambridge CB3 9EW, England \\ rah48@damtp.cam.ac.uk \\ ${ }^{2}$ Department of Mathematics and Statistics \\ University of Maryland Baltimore County \\ Baltimore, MD 21250, USA \\ guler@math.umbc.edu
}

\begin{abstract}
Self-scaled barrier functions on self-scaled cones were axiomatically introduced by Nesterov and Todd in 1994 as a tool for the construction of primal-dual long-step interior point algorithms. This paper provides firm foundations for these objects by exhibiting their symmetry properties, their close ties with the symmetry groups of their domains of definition, and subsequently their decomposition into irreducible parts and their algebraic classification theory. In the first part we recall the characterization of the family of self-scaled cones as the set of symmetric cones and develop a primal-dual symmetric viewpoint on self-scaled barriers, results that were first discovered by the second author. We then show in a short, simple proof that any pointed, convex cone decomposes into a direct sum of irreducible components in a unique way, a result which can also be of independent interest. We then proceed to showing that any self-scaled barrier function decomposes, in an essentially unique way, into a direct sum of self-scaled barriers defined on the irreducible components
\end{abstract}

Date received: December 5, 1999. Final version received: September 6, 2001. Communicated by Michael Todd. Online publication: October 19, 2001.

AMS classification: Primary, 90C25, 90C60, 52A41; Secondary, 90C06, 52A40.

Key words and phrases: Self-scaled barrier functions, Symmetric cones, Decomposition of convex cones, Jordan algebras, Universal barrier function, Interior-point methods. 
of the underlying symmetric cone. Finally, we present a complete algebraic classification of self-scaled barrier functions using the correspondence between symmetric cones and Euclidean-Jordan algebras.

\section{Introduction}

In recent years a theory of interior-point methods for linear, semidefinite, and second-order cone programming was developed within the unified framework of self-scaled conic programming. The origins of this theory can be traced to the work of Nesterov and Todd [15], [16]. Güler [4], [5] pointed out fundamental connections between the theory of self-scaled optimization and the theory of Euclidean Jordan algebras. This work was mainly concerned with barrier functions and their relations to characteristic functions of homogeneous cones, and not with interior-point algorithms. Later, Faybusovich [2] and others analyzed interior-point algorithms from the point of view of Jordan algebra machinery. The subject has since much evolved, and the Jordan algebra viewpoint has become a standard approach to self-scaled optimization. The importance of the problems which can be cast in this framework, and the fact that it is possible to develop efficient primaldual long-step interior-point methods for these problems, have contributed to the popularity of the subject in the optimization community and beyond.

In order to facilitate our exposition, we consider the following pair of convex programs in conic duality

$$
\begin{array}{ll}
\text { (P) } \quad \inf \left\langle x, s_{0}\right\rangle, & \text { (D) } \quad \inf \left\langle x_{0}, s\right\rangle, \\
x \in\left(L+x_{0}\right) \cap K, & s \in\left(L^{\perp}+s_{0}\right) \cap K^{*} .
\end{array}
$$

Here $E$ is a finite-dimensional Euclidean space equipped with an inner product $\langle\cdot, \cdot\rangle, L$ is a linear subspace of $E$, and $L^{\perp}$ is its orthogonal complement. $K$ is a regular cone, that is, it is closed, convex, solid, and pointed (it does not contain any whole lines). Moreover, $x_{0} \in \operatorname{int}(K)$ and $s_{0} \in \operatorname{int}\left(K^{*}\right)$ are fixed, where $K^{*}$ is the dual cone

$$
K^{*}:=\{s \in E:\langle x, s\rangle \geq 0, \forall x \in K\} .
$$

Interior-point algorithms can be used to solve (1.1) over any regular cone, provided one has a self-concordant barrier function $F(x)$ defined over the interior $\operatorname{int}(K)$ of $K$. Self-concordant functions are three times continuously differentiable, strictly convex, defined on an open convex domain $D_{F}$, and satisfy the technical conditions

$$
\left\{y \in E:\left\langle F^{\prime \prime}(x)(y-x), y-x\right\rangle<1\right\} \subset D_{F}, \quad \forall x \in D_{F},
$$

and

$$
1-\left\langle F^{\prime \prime}(x)(y-x), y-x\right\rangle^{1 / 2} \leq \frac{\left\langle F^{\prime \prime}(y) v, v\right\rangle^{1 / 2}}{\left\langle F^{\prime \prime}(x) v, v\right\rangle^{1 / 2}} \leq \frac{1}{1-\left\langle F^{\prime \prime}(x)(y-x), y-x\right\rangle^{1 / 2}},
$$


for all $0 \neq v \in E, x \in D_{F}$, and $y \in E$ such that $\left\langle F^{\prime \prime}(x)(y-x), y-x\right\rangle<1$. Here $F^{\prime \prime}(w)$ denotes the operator from $E$ to itself defined by $\left\langle F^{\prime \prime}(w) u, v\right\rangle=$ $\mathrm{D}^{2} F(w)[u, v]$. Note that since $F$ is convex and $\mathrm{D}^{2} F(w)$ a symmetric operator, $F^{\prime \prime}(w)$ is a positive semidefinite symmetric matrix when represented with respect to an orthogonal basis. Moreover, if the domain of definition $D_{F}$ is the interior of a regular cone, then this matrix is positive definite. Self-concordant barriers are required to satisfy the additional barrier property

$$
v:=\sup _{x \in D_{F}}\left\langle F^{\prime \prime}(x)^{-1} F^{\prime}(x), F^{\prime}(x)\right\rangle<\infty .
$$

The barrier parameter $v$ plays a central role in the complexity of interior-point methods.

It is important to note that $K^{*}, F^{\prime}, F^{\prime \prime}$, and the dual barrier $F_{*}$ to be defined later are objects whose definition depends on the inner product $\langle\cdot, \cdot\rangle$ on $E$. Whenever we use a different inner product in their definition, we introduce a different notation for distinction. The reader may consult the authoritative monographs of Nesterov and Nemirovskii [14] and of Renegar [18] for a detailed treatment of self-concordant functions, self-concordant barriers, and interior-point methods.

In linear programming, two important features of the logarithmic barrier

$$
x \mapsto-\sum_{i=1}^{n} \ln x_{i}
$$

are responsible for its wide acceptance as being superior over all other selfconcordant barriers. First, in a generic self-concordant barrier function, one has control over the behavior of the Hessians $F^{\prime \prime}(y)$ only when $y$ lies in the local ball $\left\{y:\left\langle F^{\prime \prime}(x)(y-x), y-x\right\rangle<1\right\}$, leading to short-step interior-point methods. Although these methods have a polynomial running-time guarantee, they tend to be less efficient linear programming solvers in practice than long-step interior-point methods. The theoretical basis for this latter type of algorithm is the fact that the self-concordant barrier function

$$
x \mapsto-\sum_{i=1}^{n} \ln x_{i}
$$

has additional properties which make it possible to control it in all of $\operatorname{int}(K)$. Second, in contrast to generic self-concordant barriers, the logarithmic barrier function allows one to construct primal-dual interior-point methods in which the problems (P) and (D) are solved simultaneously. In each iteration of such an algorithm, primal and dual information is exchanged in a meaningful way, which leads to improved scaling of the search directions.

In [15], Nesterov and Todd isolated two properties which are responsible for the above-mentioned advantages of the barrier $-\sum_{i=1}^{n} \ln x_{i}$ in the case of linear programming, and they generalized these properties axiomatically (see (1.4) and (1.5) below). They used the term self-scaled barrier for self-concordant barrier 
functions satisfying these conditions. Since the properties of these functions also impose certain conditions on their domain of definition, Nesterov and Todd called the closures of the corresponding domains self-scaled cones. For convenience, we recall these concepts here:

Definition 1.1. Let $K \subseteq E$ be a regular cone. A self-concordant barrier function $F: \operatorname{int}(K) \rightarrow \mathbb{R}$ is called self-scaled if $F^{\prime \prime}(x)$ is nonsingular for every $x \in K, F$ is logarithmically homogeneous, that is,

$$
F(t x)=F(x)-v \ln t, \quad \forall x \in \operatorname{int}(K), \quad t>0,
$$

and if $F$ satisfies the following two properties

$$
\begin{aligned}
F^{\prime \prime}(w) x & \in \operatorname{int}\left(K^{*}\right), & & \forall x, w \in \operatorname{int}(K), \\
F_{*}\left(F^{\prime \prime}(w) x\right) & =F(x)-2 F(w)-v, & & \forall x, w \in \operatorname{int}(K) .
\end{aligned}
$$

If $K$ allows such a barrier function, then $K$ is called a self-scaled cone.

The dual barrier $F_{*}: \operatorname{int}(K) \rightarrow \mathbb{R}$ that appears in the last axiom (1.5) is defined as

$$
F_{*}(s):=\sup \{-\langle x, s\rangle-F(x): x \in \operatorname{int}(K)\} .
$$

Proposition 3.1 in [15] shows that if $F$ is self-scaled with barrier parameter $v$, then $F_{*}$ is a self-scaled barrier with the same parameter $v$. Theorems 3.1 and 3.2 in [15] state that (1.4) can be strengthened to the following result.

Theorem 1.2. If $x \in \operatorname{int}(K)$ and $s \in \operatorname{int}\left(K^{*}\right)$, then there exists a unique point $w \in \operatorname{int}(K)$ such that $F^{\prime \prime}(w) x=s$. Moreover, for all $w \in \operatorname{int}(K), F^{\prime \prime}(w)$ $(K)=K^{*}$.

See also the end of this section for further remarks regarding this result. The point $w$ is called the scaling point of $x$ and $s$. Using rather elementary tools, Rothaus [19] proved a number of results which are useful in Section 3 of this paper. Two key results are [19, Theorem 3.12, Cor. 3.15, p. 205]. These results imply Theorem 1.2 for the universal barrier function, a special self-concordant barrier function defined by Nesterov and Nemirovskii [14] which is further discussed below. Theorem 1.2 was found by Nesterov and Todd independently and provides an extension of Rothaus's result to general self-scaled barriers. It can be shown that, when suitably modified, all results of Section III in [19] can be extended to general self-scaled barriers.

Nesterov and Todd [15], [16] demonstrated that self-scaled barrier functions can indeed be used to develop various primal-dual long-step interior-point methods for linear optimization over self-scaled cones, in particular, for semidefinite programming and for convex quadratic programming with convex quadratic constraints. 
Inspired by the paper of Vinberg [21], Güler [5] related the universal barrier function of Nesterov and Nemirovskii [14] to the characteristic function introduced by Koecher [11]. For a regular cone $K \subset E$ and an appropriately chosen constant $c$, the universal barrier is defined by

$$
U_{K}(x):=c \ln \operatorname{vol}\{s \in E:\langle s, y-x\rangle \leq 1, \forall y \in K\},
$$

where vol denotes the canonical Lebesgue measure on $(E,\langle\cdot, \cdot\rangle)$. The characteristic function of $K$ is defined as

$$
\varphi_{K}(x):=\int_{K^{*}} e^{-\langle x, y\rangle} d y .
$$

Güler showed that there exist constants $\gamma>0$ and $\delta$ such that

$$
U_{K}(x)=\gamma \ln \varphi_{K}(x)+\delta,
$$

and that interiors of self-scaled cones and so-called symmetric cones (see Definition 1.3 below) are the same class of objects. Through this connection between previously distinct ideas, the concepts of homogeneous cones, homogeneous selfdual cones (or symmetric cones), Euclidean Jordan algebras, and Siegel domains, as well as the classification theory of symmetric cones and Euclidean Jordan algebras, known to mathematicians since 1960 and 1934, respectively, became important tools in the interior-point literature. The interested reader can find a complete treatment of these classification results in the book by Faraut and Korányi [1]. See also [11] for a different treatment of some of the same topics.

Because of their importance for this paper, we recall some of the concepts mentioned above.

Definition 1.3. Let $K \subseteq E$ be a regular cone. The automorphism group of $K$ is the set of all nonsingular linear maps $A: E \rightarrow E$ that leave $K$ invariant, i.e.,

$$
\operatorname{Aut}(K):=\{A \in \mathrm{GL}(E): A(K)=K\} .
$$

The cone $K$ is called homogeneous if $\operatorname{Aut}(K)$ acts transitively on $\operatorname{int}(K)$, that is, given arbitrary points $x, y \in \operatorname{int}(K)$, there exists a map $A \in \operatorname{Aut}(K)$ such that $A x=y$. The cone $K$ is called self-dual if $E$ can be endowed with an inner product such that $K^{*}=K$ where $K^{*}$ is defined with respect to this inner product, see (1.2). The cone $K$ is called symmetric if $K$ is both homogeneous and self-dual.

As mentioned earlier, symmetric cones have been fully classified in the theory of Euclidean Jordan algebras, see [11], [1] and the references therein. According to this theory, each symmetric cone has a unique decomposition into a direct sum of elementary building blocks, so-called irreducible symmetric cones, of which there exist only five types. Three examples of symmetric cones are of particular interest to the optimization community: The nonnegative orthant $\mathbb{R}_{+}^{n}$ 
(this is in fact the direct sum of $n$ irreducible symmetric cones which are halflines), the cone $\Sigma_{n}^{+}$of $n \times n$ symmetric, positive semidefinite matrices over the real numbers, and the Lorentz cone $\left\{\left(\begin{array}{l}\tau \\ x\end{array}\right) \in \mathbb{R}^{n+1}: \tau \geq\|x\|_{2}\right\}$. The general self-scaled conic optimization problems associated with these cones are, respectively, linear programming, semidefinite programming, and second-order cone programming. The latter can be seen as a reformulation of convex quadratic programming with convex quadratic constraints. Considering more general symmetric cones, one can treat linear optimization problems with mixed linear, semidefinite, and convex quadratic constraints in a single unified framework.

Motivated by [5], and by the fact that only a small number of examples of selfscaled barrier functions were explicitly known, Hauser developed a decomposition theory and a partial algebraic classification for self-scaled barrier functions in a chapter of his thesis [8]. These results were later announced in a technical report [9]. The paper left the open problem of proving a conjecture according to which all selfscaled barrier functions defined on irreducible symmetric cones are rotationally invariant (isotropic). In [9] it was shown that if this conjecture were true, then all self-scaled barriers could be algebraically classified. In [10], Hauser proved this conjecture for the special case of the positive semidefinite cone. The key result in the proof was Proposition 3.3, which was derived from first principles but later turned out to be a close relative of a more general result by Koecher [11, Theorem 4.9(b), pp. 88-89], which applies to all irreducible symmetric cones. Using this result, Lim [12], Schmieta [20], and Güler [6], all independently of each other, settled the above-mentioned conjecture in the general case.

The rest of the paper is organized as follows. In Section 2 we reconsider selfscaled cones and self-scaled barriers from a symmetric point of view. Section 3 is devoted to certain properties of self-scaled barriers which link self-scaled barriers to the symmetry group of their domain of definition. These results are needed in later sections. In Section 4, we show that any pointed, convex cone has a unique decomposition into a direct sum of irreducible components. This result, of which we managed to locate only technically more involved generalizations in the literature, may be of independent interest. We therefore include a simple proof. We then use this decomposition result to show that any self-scaled barrier defined on a symmetric cone $K$ decomposes in an essentially unique way into a direct sum of self-scaled barriers defined on the irreducible components of $K$, which also shows that the irreducible components are symmetric cones themselves. This decomposition reduces the problem of classifying self-scaled barriers to the case where the domain of definition is irreducible, a problem we solve in Section 5. Theorem 5.5 constitutes the main and final result of this paper. We thus present all the essential elements of the theory of self-scaled barrier functions in a single document.

The following basic properties of $v$ self-concordant logarithmically homogeneous barrier functions and their duals will be used frequently in later sections. These properties are easy consequences of logarithmic homogeneity alone, see [14] or [15]. 
Proposition 1.4. Let $F$ be a $v$ self-concordant logarithmically homogeneous barrier function on the regular cone $K \subset E$, and let $x \in \operatorname{int}(K), s \in \operatorname{int}\left(K^{*}\right)$. Then:

(i) $-F^{\prime}(x)=F^{\prime \prime}(x) x \in \operatorname{int}\left(K^{*}\right)$;

(ii) $F^{(k)}(t x)=t^{-k} F^{(k)}(x), \forall t>0$;

(iii) $-F_{*}^{\prime}\left(-F^{\prime}(x)\right)=x$;

(iv) $F_{*}^{\prime \prime}\left(-F^{\prime}(x)\right)=F^{\prime \prime}(x)^{-1}$;

(v) $\left\langle x,-F^{\prime}(x)\right\rangle=v$;

(vi) $F_{*}\left(-F^{\prime}(x)\right)=-v-F(x)$; and

(vii) $F(x)+F_{*}(s) \geq-v-v \log v-v \log \langle x, s\rangle$.

Using this proposition, it is easy to see that the last part of Theorem 1.2 follows from (1.4) and from [15, Eq. (3.2)], which we reproduce here for convenience,

$$
F^{\prime \prime}(x)=F^{\prime \prime}(w) F_{*}^{\prime \prime}\left(F^{\prime \prime}(w) x\right) F^{\prime \prime}(w) .
$$

Indeed, it is sufficient to show that $F^{\prime \prime}(w)(\operatorname{int}(K))=\operatorname{int}\left(K^{*}\right)$, and (1.4) shows that the left-hand side is contained in the right-hand side. For the reverse, any $y \in \operatorname{int}\left(K^{*}\right)$ can be written as $F^{\prime \prime}(x) x$ where $x:=-F_{*}^{\prime}(y)$, and then (1.8) shows that $y=F^{\prime \prime}(w) z$, where $z=F_{*}^{\prime \prime}\left(F^{\prime \prime}(w) x\right) F^{\prime \prime}(w) x$ lies in int $(K)$ by (1.4) applied to $F$ and to $F_{*}$. Formula (1.8) also reappears in Lemma 2.3, where we give a proof of this important identity.

\section{A Symmetric View on Self-Scaledness}

In this section we undertake a study of self-scaled cones and barrier functions while emphasizing their symmetry properties in a duality-theoretic sense.

Let $F$ be a self-scaled barrier function on a regular cone $K$ in a finite-dimensional Euclidean space $E$ equipped with an inner product $\langle\cdot, \cdot\rangle$. With a given arbitrary point $e \in \operatorname{int}(K)$ we associate an inner product

$$
\langle u, v\rangle_{e}:=\left\langle F^{\prime \prime}(e) u, v\right\rangle .
$$

Note that the dual cone $K^{*}$, the tensors $F^{\prime}, F^{\prime \prime}$, and the dual barrier $F_{*}$ depend on the choice of the inner product $\langle\cdot, \cdot\rangle$. Choosing the inner product $\langle\cdot, \cdot\rangle_{e}$ thus defines a different set of objects which we denote by $K_{e}^{*}, F_{e}^{\prime}, F_{e}^{\prime \prime}$, and $\left(F_{*}\right)_{e}$, respectively. The following result is due to Güler [4].

Theorem 2.1. The cone $K$ is symmetric, and $F$ is self-scaled under $\langle\cdot, \cdot\rangle_{e}$. Moreover, $F_{e}^{\prime \prime}(e)=I$.

Proof. We have

$$
\begin{aligned}
K_{e}^{*} & :=\left\{y:\langle x, y\rangle_{e} \geq 0, \forall x \in K\right\}=\left\{y:\left\langle F^{\prime \prime}(e) x, y\right\rangle \geq 0, \forall x \in K\right\} \\
& =\left\{y:\langle z, y\rangle \geq 0, \forall z \in K^{*}\right\}=\left(K^{*}\right)^{*}=K,
\end{aligned}
$$


where the third equality follows from Theorem 1.2. Note that

$$
\left\langle F^{\prime \prime}(x) u, v\right\rangle=D^{2} F(x)[u, v]=\left\langle F_{e}^{\prime \prime}(x) u, v\right\rangle_{e}=\left\langle F^{\prime \prime}(e) F_{e}^{\prime \prime}(x) u, v\right\rangle
$$

yields $F^{\prime \prime}(x)=F^{\prime \prime}(e) F_{e}^{\prime \prime}(x)$, or

$$
F_{e}^{\prime \prime}(x)=F^{\prime \prime}(e)^{-1} F^{\prime \prime}(x) .
$$

Theorem 1.2 implies that $F_{e}^{\prime \prime}(x)(K)=F^{\prime \prime}(e)^{-1} F^{\prime \prime}(x)(K)=F^{\prime \prime}(e)^{-1}\left(K^{*}\right)=K$, so that $F_{e}^{\prime \prime}(x) \in \operatorname{Aut}(K)$. Theorem 1.2 also shows that, given any two points $u, v \in \operatorname{int}(K)$, we can find a (unique) point $z \in \operatorname{int}(K)$ such that $F^{\prime \prime}(z) u=$ $F^{\prime \prime}(e) v \in K^{*}$. Therefore, $F_{e}^{\prime \prime}(x)(u)=v$, which shows that the set of linear operators $\left\{F_{e}^{\prime \prime}(x): x \in \operatorname{int}(K)\right\}$ acts transitively on $\operatorname{int}(K)$. Hence, $K$ is a symmetric cone.

For the second assertion, note that if $s \in K_{e}^{*}=K$, then

$$
\begin{aligned}
\left(F_{*}\right)_{e}(s) & :=\sup _{x \in K}\left\{-\langle x, s\rangle_{e}-F(x)\right\} \\
& =\sup _{x \in K}\left\{-\left\langle x, F^{\prime \prime}(e) s\right\rangle-F(x)\right\}=F_{*}\left(F^{\prime \prime}(e) s\right) .
\end{aligned}
$$

For $x, z \in \operatorname{int}(K)$, we thus have

$$
\left(F_{*}\right)_{e}\left(F_{e}^{\prime \prime}(z) x\right)=F_{*}\left(F^{\prime \prime}(e) F_{e}^{\prime \prime}(z) x\right)=F_{*}\left(F^{\prime \prime}(z) x\right)=F(x)-2 F(z)-v,
$$

where the second and last equalities follow from (2.1) and (1.5), respectively. Consequently, $F$ is self-scaled under $\langle\cdot, \cdot\rangle_{e}$.

The last statement follows from (2.1).

Remark 2.2. From here on we may assume without loss of generality that the inner product $\langle\cdot, \cdot\rangle$ equals $\langle\cdot, \cdot\rangle_{e}$ for some point $e \in \operatorname{int}(K)$, i.e., we may assume that $K$ is symmetric by virtue of Theorem 2.1. Note that Theorem 1.2 implies that $e$ is the unique point with the property $F^{\prime \prime}(e)=I$. In fact, instead of assuming $\langle\cdot, \cdot\rangle=\langle\cdot, \cdot\rangle_{e}$, it would be equivalent to assume that $\langle\cdot, \cdot\rangle$ is chosen so that $K$ is symmetric, and it will later follow from Lemma 3.4 that there exists a unique point $e$ such that $F^{\prime \prime}(e)=I$ and $\langle\cdot, \cdot\rangle=\langle\cdot, \cdot\rangle_{e}$. Together with (1.5) our assumption implies that

$$
F_{*}(x)=F(x)-2 F(e)-v=F(x)+\text { const, }
$$

and invoking (1.5) once more this implies the identity

$$
F\left(F^{\prime \prime}(w) x\right)=F(x)-2 F(w)+2 F(e), \quad \forall x, w \in \operatorname{int}(K) .
$$

Note that (2.3) is a criterion that involves only the primal barrier $F$. Indeed, this identity allows one to characterize self-scaled barrier functions without invoking $F_{*}$, see Lemma 2.5 below. Changing a barrier function by an additive constant is of no real consequence, as interior-point methods rely on gradient and Hessian information. Therefore, we could assume that $F(e)=-v / 2$ so that $F_{*}=F$. We will not make this assumption, but it shows that we no longer need to distinguish between primal and dual quantities conceptually, between $F$ and $F_{*}$, the primal and dual scaling points, and so forth. 
We next prove a property of the Hessian $F^{\prime \prime}(w)$ which will become an essential tool for our classification of self-scaled barriers. For all $y \in \operatorname{int}(K)$ let us define

$$
P(y):=F^{\prime \prime}(y)^{-1} \text {. }
$$

Lemma 2.3. For all $x, w \in \operatorname{int}(K)$ it is true that

$$
P(P(w) x)=P(w) P(x) P(w) .
$$

Proof. Let us define $z=P(w) x$. Equation (2.3) implies that for any $h \in E$ and all $t$ sufficiently small we have $F\left(F^{\prime \prime}(w)(z+t h)\right)=F(z+t h)-2 F(w)+2 F(e)$. Differentiating this equation twice with respect to $t$ and then setting $t$ to zero one gets

$$
D^{2} F\left(F^{\prime \prime}(w) z\right)\left[F^{\prime \prime}(w) h, F^{\prime \prime}(w) h\right]=D^{2} F(z)[h, h],
$$

or $\left\langle F^{\prime \prime}(x) F^{\prime \prime}(w) h, F^{\prime \prime}(w) h\right\rangle=\left\langle F^{\prime \prime}(z) h, h\right\rangle$. Thus, $F^{\prime \prime}(w) F^{\prime \prime}(x) F^{\prime \prime}(w)=F^{\prime \prime}(z)$ $=F^{\prime \prime}(P(w) x)$, and (2.4) follows.

In the proof above, we need only the weaker condition $F\left(F^{\prime \prime}(w) x\right)=F(x)+$ $c(w)$, where $c(\cdot)$ is a function defined on $\operatorname{int}(K)$. However, Lemma 2.5 below shows that this is equivalent to (2.3). Equation (2.4) is a symmetric version of formula (3.2) from [15], see also (1.8) above. In accordance with the established tradition in the theory of Jordan algebras we call (2.4) the fundamental formula.

Remark 2.4. Petersson's work [17] and the fundamental formula suggest that $F$ might define a natural Jordan algebra. Güler [6] and Schmieta [20] independently proved that this is indeed the case, a fact which was used by Schmieta to classify self-scaled barriers. However, the natural Jordan algebra connected to $F$ had already been discovered by McCrimmon in his thesis [13], even without the assumption of the convexity of $F$. His proof in turn was a generalization of Koecher's ideas [11] on $\omega$-domains. Reading both works is instructive in delineating the role of convexity.

The following result provides an alternative definition of self-scaled barrier functions.

Lemma 2.5. Let $K$ be a regular, self-dual cone. A logarithmically homogeneous self-concordant barrier function $F$ defined on $\operatorname{int}(K)$ is self-scaled if and only if

$$
\begin{array}{clrl}
F^{\prime \prime}(w) x & \in \operatorname{int}(K), & \forall x, w \in \operatorname{int}(K), \\
F\left(F^{\prime \prime}(w) x\right) & =F(x)+c(w), & \forall x, w \in \operatorname{int}(K),
\end{array}
$$

where $c(w)$ is a function defined on $\operatorname{int}(K)$.

Proof. Since $K$ is self-dual, (2.5) is equivalent to axiom (1.4). If $F$ is selfscaled, then (2.6) follows from (2.3). For the converse we repeat the argument 
from the proof of [15, Theorem 3.2]: let us assume that $F$ satisfies (2.6). Let $x, s \in \operatorname{int}(K)$ be arbitrary points. We claim that there exists a point $w \in \operatorname{int}(K)$ such that $F^{\prime \prime}(w) x=s$. Toward proving this claim, we consider the optimization problem $\min \left\{\left\langle z^{*}, x\right\rangle:\langle z, s\rangle=1, z \in \operatorname{int}(K)\right\}$, where $z^{*}=-F^{\prime}(z)$. It is well known that the feasible region is bounded, see [1, Cor. I.1.6, p. 4]. We have

$$
F(x)+F_{*}\left(z^{*}\right) \geq-v-v \log v-v \log \left\langle z^{*}, x\right\rangle,
$$

see Proposition 1.4(vii), and $F(z)+F_{*}\left(z^{*}\right)=-v$, see Proposition 1.4(vi). Therefore,

$$
F(x)-F(z) \geq-v \log v-v \log \left\langle z^{*}, x\right\rangle .
$$

This implies that the objective function of the optimization problem goes to infinity as $z$ approaches the boundary of the feasible region, and thus the optimization problem has a minimizer $\hat{z} \in \operatorname{int}(K)$ satisfying $F^{\prime \prime}(\hat{z}) x=\lambda s$ for some scalar $\lambda$. Since $F^{\prime \prime}(\hat{z}) x, s \in \operatorname{int}(K)$, we have $\lambda>0$. The point $w=\sqrt{\lambda} \hat{z}$ satisfies $F^{\prime \prime}(w) x=s$, see Proposition 1.4(ii). This proves our claim.

Next, we claim that

$$
c(w)=-2 F(w)+2 F(e) .
$$

Let $u \in \operatorname{int}(K)$ be a point satisfying $F^{\prime \prime}(u) w=e$. The fundamental formula (2.4) is a consequence of (2.6) and gives $F^{\prime \prime}(u) P(w) F^{\prime \prime}(u)=I$ or, equivalently, $F^{\prime \prime}(w)=F^{\prime \prime}(u)^{2}$. From (2.6), we obtain

$$
F(e)+c(w)=F\left(F^{\prime \prime}(w) e\right)=F\left(F^{\prime \prime}(u)^{2} e\right)=F(e)+2 c(u),
$$

or $c(w)=2 c(u)$. Equation (2.6) also implies that

$$
F(e)=F\left(F^{\prime \prime}(u) w\right)=F(w)+c(u)=F(w)+\frac{1}{2} c(w),
$$

hence proving the claim.

Using logarithmic homogeneity alone one can prove that $F_{*}\left(w^{*}\right)=-v-F(w)$ where $w^{*}:=-F^{\prime}(w)$ (see Proposition 1.4(vi)). Proposition 1.4(iii) shows that the mapping $w \mapsto w^{*}$ is involutive, that is, $w^{* *}=w$. These imply $F_{*}(w)=$ $F_{*}\left(w^{* *}\right)=-v-F\left(w^{*}\right)$. Since $F^{\prime \prime}(w) w=w^{*}$ by Proposition 1.4(i), we have

$$
-v-F_{*}(w)=F\left(w^{*}\right)=F\left(F^{\prime \prime}(w) w\right)=F(w)-2 F(w)+2 F(e),
$$

which is to say that $F_{*}(w)=F(w)-2 F(e)-v$. This implies

$$
F_{*}\left(F^{\prime \prime}(w) x\right)=F\left(F^{\prime \prime}(w) x\right)-2 F(e)-v=F(x)-2 F(w)-v,
$$

where the last equality follows from (2.6) and (2.7). This concludes the proof.

Note that together with (2.2), Lemma 2.5 implies that we can replace axiom (1.5) of the original definition of a self-scaled barrier function by the requirement $F_{*}\left(F^{\prime \prime}(w) x\right)=F(x)+c(w)$ for some function $c$ : $\operatorname{int}(K) \rightarrow \mathbb{R}$. This fact is already known, see [18]. 


\section{Group-Theoretic Aspects of Self-Scaledness}

In this section we explore the relationship between the Hessians of self-scaled barrier functions and the symmetry group of their domain of definition. Though we present these results primarily for the purposes of later sections they are also of independent interest.

The universal barrier function $U(x)$ defined in (1.7) plays an important role in the context of this section. The choice of the inner product $\langle\cdot, \cdot\rangle$ used in the definition of the characteristic function $\varphi_{K}(x)$ via (1.6) is irrelevant, since $\ln \varphi_{K}$ changes only by an additive constant under a change of $\langle\cdot, \cdot\rangle$. It is known that the universal barrier function $U(x)$ is self-scaled, see Equation (13) and Theorem 4.4 in Güler [5]. For all $x \in \operatorname{int}(K)$ let

$$
Q(x):=U^{\prime \prime}(x)^{-1}
$$

and let $f \in \operatorname{int}(K)$ be the point characterized by the equation

$$
Q(f)=\mathrm{I} .
$$

Remark 3.1. It follows from Theorem 1.2 that $f$ is unique. The existence of such a point is also well known, see, for example, page 17 of [1].

The point $f$ is the "unit" associated with the self-scaled barrier $U(x)$, see [1, Prop. I.3.5, p. 14], and it is also the unit of the Jordan algebra associated with $U$.

The following lemma is Theorem 3.17, pp. 205-206 in [19]. We include a short proof of this result because these ideas play an important role in later sections. See also [1, Prop. I.4.3, p. 18] for a different approach to proving this result.

Lemma 3.2. The orthogonal subgroup $O(\operatorname{Aut}(K)) \subseteq \operatorname{Aut}(K)$ coincides with the stabilizer group at $f$, that is,

$$
O(\operatorname{Aut}(K))=\{H \in \operatorname{Aut}(K): H f=f\} .
$$

Proof. If $A \in \operatorname{Aut}(K)$, then

$$
D^{2} U(A f)[A h, A h]=D^{2} U(f)[h, h]
$$

for every vector $h \in E$. That is, $A^{*} Q(A f)^{-1} A=I$, or $Q(A f)=A A^{*}$, see, for example, [5, Eq. (11)]. This shows that $A$ is orthogonal if and only if $I=Q(A f)$. The uniqueness of $f$ implies that this condition is equivalent to $A f=f$.

Next we note that the elements of $\operatorname{Aut}(K)$ have a unique polar decomposition, see [19, Theorem 3.18, p. 206]. For the sake of completeness we give a short proof. 
Lemma 3.3. Let $A \in \operatorname{Aut}(K)$. There exists a unique vector $u \in \operatorname{int}(K)$ and $a$ unique orthogonal cone automorphism $H \in O(\operatorname{Aut}(K))$ such that

$$
A=Q(u) H .
$$

Proof. By virtue of Theorem 1.2, there exists a unique point $u \in \operatorname{int}(K)$ such that $Q(u) f=A f$. Then $H:=Q(u)^{-1} A$ satisfies $H f=Q(u)^{-1} A f=f$, which implies that $H$ is orthogonal by Lemma 3.2. Since $H$ is orthogonal and $Q(u)$ is symmetric, $A=Q(u) H$ is indeed a polar decomposition of $A$.

Suppose now that $A=Q_{1} H_{1}=Q_{2} H_{2}$ where $Q_{i}$ is symmetric and $H_{i}$ is orthogonal, $i=1$, 2. Then, $H:=Q_{2}^{-1} Q_{1}=H_{2} H_{1}^{-1}$ is orthogonal, and we have $I=H H^{*}=Q_{2}^{-1} Q_{1}^{2} Q_{2}^{-1}$, or $Q_{2}^{2}=Q_{1}^{2}$. Since $Q_{1}$ and $Q_{2}$ are symmetric, we have $Q_{1}=Q_{2}$ and $H_{1}=H_{2}$.

The following result will play a key role in Section 5 where we classify selfscaled barriers.

Lemma 3.4. The sets of inverse Hessians of $F$ and $U$ coincide, that is,

$$
\{P(v): v \in \operatorname{int}(K)\}=\{Q(u): u \in \operatorname{int}(K)\},
$$

and for all $x \in \operatorname{int}(K)$ it is true that

$$
P(x)=Q\left(Q(x)^{1 / 2} e^{-1}\right)=Q(x)^{1 / 2} Q(e)^{-1} Q(x)^{1 / 2},
$$

where $e^{-1} \in \operatorname{int}(K)$ is characterized by the equation $Q\left(e^{-1}\right)=Q(e)^{-1}$.

The point $e^{-1}$ is the inverse of $e$ in the Jordan algebra associated with $U(x)$, see e.g., [1]. Note that Proposition 1.4(iv) shows that $e^{-1}=-U^{\prime}(e)$, also proving the existence of such a point.

Proof. If $v \in \operatorname{int}(K)$, then Theorem 1.2 and Lemma 3.3 imply that we can write $P(v)=Q(u) H$ for some $u \in \operatorname{int}(K)$ and $H \in O(\operatorname{Aut}(K))$. By the uniqueness of the polar decompositions $P(v)=P(v) I$ and $P(v)=Q(u) H$ we must have that $P(v)=Q(u)$. Thus,

$$
\{P(v): v \in \operatorname{int}(K)\} \subseteq\{Q(u): u \in \operatorname{int}(K)\} .
$$

Conversely, let $u \in \operatorname{int}(K)$. By Theorem 1.2, there exists a point $v \in \operatorname{int}(K)$ such that $P(v) f=Q(u) f$. But this implies that $Q(u)^{-1} P(v) f=f$. By virtue of Lemma $3.2 H:=Q(u)^{-1} P(v)$ is therefore orthogonal. This means that $P(v)$ has the polar decompositions $P(v)=P(v) I$ and $P(v)=Q(u) H$. The uniqueness part of Lemma 3.2 then implies that $Q(u)=P(v)$ and $H=I$. This proves the first statement of the lemma.

Now let $x \in \operatorname{int}(K)$ and define $u$ by $x=Q(u) f$, see Theorem 1.2. We have

$$
Q(x)=Q(Q(u) f)=Q(u) Q(f) Q(u)=Q(u)^{2},
$$


where the second equality follows from the fundamental formula (2.4). In a similar vein, taking the first part of this lemma into account, we obtain

$$
P(x)=P(Q(u) f)=Q(u) P(f) Q(u) .
$$

These two equations imply that $Q(u)=Q(x)^{1 / 2}$ and

$$
P(x)=Q(x)^{1 / 2} P(f) Q(x)^{1 / 2} .
$$

In particular, setting $x=e$ yields $I=Q(e)^{1 / 2} P(f) Q(e)^{1 / 2}$, that is, $P(f) Q(e)=$ $I$, and $P(f)=Q(e)^{-1}=Q\left(e^{-1}\right)$. The lemma follows, since this implies that

$$
P(x) \stackrel{(3.2)}{=} Q(u) Q\left(e^{-1}\right) Q(u) \stackrel{\text { Lem } 2.3}{=} Q\left(Q(u) e^{-1}\right)=Q\left(Q(x)^{1 / 2} e^{-1}\right) .
$$

Although it does not have a direct bearing on later results, the following proposition already shows that the self-scaled barrier function $F$ is intimately connected to the universal barrier function.

Proposition 3.5. There exist constants $\alpha_{1}>0$ and $\alpha_{2}$ such that

$$
U(x)=\alpha_{1} \ln \operatorname{det} F^{\prime \prime}(x)+\alpha_{2} .
$$

Proof. From (3.1) we see that $\operatorname{det} P(x)=\operatorname{det} Q(e)^{-1} \operatorname{det} Q(x)$, implying that $\operatorname{det} F^{\prime \prime}(x)=\kappa_{1} \operatorname{det} U^{\prime \prime}(x)$ for some constant $\kappa_{1}>0$. Theorem 4.4 in [5] shows that the function $u(x)=\ln \varphi_{K}(x)$ satisfies the equation $u(x)=\kappa_{2}+\frac{1}{2} \ln \operatorname{det} u^{\prime \prime}(x)$ for some constant $\kappa_{2}$. These facts combined with (1.7) imply the proposition.

\section{Decomposition of Cones and Barrier Functions}

In this section, we prove two related results. Recall that a cone is called pointed if it does not contain any whole lines. First, we show that any pointed, convex cone decomposes into a direct sum of indecomposable or irreducible components in a unique fashion. This theorem is also of independent interest, and it is essentially a special case of Corollary 1 in Gruber [3], the earliest occurrence of this result we could locate in the literature, though it may have been derived several times independently. Gruber's original result addresses a more general affine setting which renders his proof more technically involved. Therefore, we include a simple and accessible proof. Second, we use this decomposition to write any self-scaled barrier function defined on the interior of a symmetric cone $K$ as a direct sum of self-scaled barriers defined on the irreducible components of $K$.

Recall that the Minkowski sum of a set $\left\{A_{i}\right\}_{i=1}^{k}$ of subsets of $E$ is defined as

$$
A_{1}+\cdots+A_{k}:=\left\{\sum_{i=1}^{k} x_{i}: x_{i} \in A_{i}\right\} .
$$


If all of the $A_{i}$ are linear subspaces $\{0\} \neq E_{i} \subseteq E$ which satisfy $E=E_{1}+\cdots+E_{k}$ and $E_{i} \cap\left(\sum_{j \neq i} E_{j}\right)=\{0\}$, then we say that the sum $E=E_{1}+\cdots+E_{m}$ is direct and write

$$
E=E_{1} \oplus E_{2} \oplus \cdots \oplus E_{m}
$$

Definition 4.1. Let $K \subseteq E$ be a pointed, convex cone. $K$ is called decomposable if there exist cones $\left\{K_{i}\right\}_{i=1}^{m}, m \geq 2$, such that $K=K_{1}+\cdots+K_{m}$, where each $K_{i}, i=1, \ldots, m$, lies in a linear subspace $E_{i} \subset E$, and where the spaces $\left\{E_{i}\right\}_{i=1}^{m}$ decompose $E$ into a direct sum $E=E_{1} \oplus E_{2} \oplus \cdots \oplus E_{m}$. Each $K_{i}$ is called a direct summand of $K$, and $K$ is called the direct sum of the $\left\{K_{i}\right\}$. We write

$$
K=K_{1} \oplus K_{2} \oplus \cdots \oplus K_{m}
$$

to denote this relationship between $K$ and $\left\{K_{i}\right\}_{i=1}^{m} . K$ is called indecomposable or irreducible if it cannot be decomposed into a nontrivial direct sum.

Let us define $\hat{E}_{i}:=\bigoplus_{j \neq i} E_{j}$ and $\hat{K}_{i}:=\bigoplus_{j \neq i} K_{j}$. If $K$ is the direct sum (4.1), then every $x \in K$ has a unique representation $x=x_{1}+\cdots+x_{m}$ with $x_{i} \in K_{i} \subseteq E_{i}$. Thus, $x_{i}=\pi_{E_{i}} x$, where $\pi_{E_{i}}$ is the projection of $E$ onto $E_{i}$ along $\hat{E}_{i}$. Also, since $0 \in K_{i}$, we have $K_{i}=K_{i}+\sum_{j \neq i}\{0\} \subseteq \sum_{j=1}^{m} K_{j}=K$. Therefore,

$$
\pi_{E_{i}} K=K_{i} \subseteq K \text {. }
$$

This implies that $K_{i}=\pi_{E_{i}} K$ is a convex cone. Similarly, we have

$$
\left(\mathrm{I}-\pi_{E_{i}}\right) K=\pi_{\hat{E}_{i}} K=\hat{K}_{i} \subseteq K .
$$

We first prove a useful technical result:

Lemma 4.2. Let $K$ be a pointed, convex cone which decomposes into the direct sum (4.1). If $x \in K_{i}$ is a sum $x=x_{1}+\cdots+x_{k}$ of elements $x_{j} \in K$, then each $x_{j} \in K_{i}$.

Proof. We have $0=\pi_{\hat{E}_{i}} x=\pi_{\hat{E}_{i}} x_{1}+\cdots+\pi_{\hat{E}_{i}} x_{k}$. Each term $\hat{x}_{j}:=\pi_{\hat{E}_{i}} x_{j} \in$ $\hat{K}_{i} \subseteq K$, therefore we have $\hat{x}_{j} \in K$ and $-\hat{x}_{j}=\sum_{l \neq j} \hat{x}_{l} \in K$. Since $K$ contains no lines it must be true that $\hat{x}_{j}=0$, that is, $x_{j}=\pi_{E_{i}} x_{j} \in K_{i}, j=1, \ldots, k$.

Theorem 4.3. Let $K \subseteq E$ be a decomposable, pointed, convex cone. The irreducible decompositions of $K$ are identical modulo indexing, that is, the set of cones $\left\{K_{i}\right\}_{i=1}^{m}$ is unique. Moreover, the subspaces $E_{i}$ corresponding to the nonzero cones $K_{i}$ are also unique. In particular, if $K$ is solid, then all the cones $K_{i}$ are nonzero and the subspaces $E_{i}$ are unique. 
Proof. Suppose that $K$ admits two irreducible decompositions

$$
K=\bigoplus_{i=1}^{m} K_{i} \subseteq \bigoplus_{i=1}^{m} E_{i}
$$

and

$$
K=\bigoplus_{j=1}^{q} C_{j} \subseteq \bigoplus_{j=1}^{q} F_{j}
$$

Note that each nonzero summand in either decomposition of $K$ must lie in $\operatorname{span}(K)$ and that the subspace corresponding to each zero summand must be one-dimensional for, otherwise, the summand would be decomposable. This implies that the number of zero summands in both decompositions is $\operatorname{codim}(\operatorname{span}(K))$. We may thus concentrate our efforts on $\operatorname{span}(K)$, that is, we can assume that $K$ is solid and that all the summands of both decompositions of $K$ are nonzero. By (4.1), each $x \in C_{j} \subseteq K$ has a unique representation $x=x_{1}+\cdots+x_{m}$ where $x_{i}=$ $\pi_{E_{i}} x \in K_{i} \subseteq K$. Also, Lemma 4.2 implies that $x_{i} \in C_{j}$, and hence $x_{i} \in K_{i} \cap C_{j}$. Consequently, every $x \in C_{j}$ lies in the set $\left(K_{1} \cap C_{j}\right)+\cdots+\left(K_{m} \cap C_{j}\right)$. Conversely, we have $K_{i} \cap C_{j} \subseteq C_{j}$, implying that $\left(K_{1} \cap C_{j}\right)+\cdots+\left(K_{m} \cap C_{j}\right) \subseteq C_{j}$. Therefore, it is true that

$$
C_{j}=\left(K_{1} \cap C_{j}\right)+\cdots+\left(K_{m} \cap C_{j}\right) .
$$

Note that $K_{i} \cap C_{j} \subseteq E_{i} \cap F_{j}, F_{j}=\left(E_{1} \cap F_{j}\right)+\cdots+\left(E_{m} \cap F_{j}\right)$, and that the intersection of any two distinct summands in the last sum is the trivial subspace $\{0\}$. The above decompositions of $F_{j}$ and $C_{j}$ are therefore direct sums. Since $C_{j}$ is indecomposable, exactly one of the summands in the decomposition of $C_{j}$ is nontrivial. Thus, $C_{j}=K_{i} \cap C_{j}$, and hence $C_{j} \subseteq K_{i}$ for some $i$. Arguing symmetrically, we also have $K_{i} \subseteq C_{l}$ for some $l$, implying that $C_{j} \subseteq C_{l}$. Therefore, $j=l$ or else $C_{j} \subseteq F_{j} \cap F_{l}=\{0\}$, contradicting our assumption above. This shows that $C_{j}=K_{i}$. The theorem is proved by repeating the above arguments for the cone $\hat{K}_{i}=\bigoplus_{k \neq i} K_{k}=\bigoplus_{l \neq j} C_{l}$.

Next, we show that self-scaled barrier functions have irreducible decompositions as well. As a side result, we obtain the information that the irreducible components of a symmetric cone are symmetric, a result which is of course wellknown from the classification theory of symmetric cones, see [1]. Let $F$ be defined on $\operatorname{int}(K)$ where $K$ is a symmetric cone with irreducible decomposition (4.1). For $i=1, \ldots, m$, let $F_{i}$ be a function defined on $\operatorname{ri}\left(K_{i}\right)$, the relative interior of $K_{i}$ in $E$. If $F(x)=\sum_{i=1}^{m} F_{i}\left(x_{i}\right)$ for every $x=\sum_{i=1}^{m} x_{i} \in \bigoplus_{i=1}^{m} \operatorname{ri}\left(K_{i}\right)=\operatorname{int}(K)$, then we say that $F$ is the direct sum of the $F_{i}$ and write

$$
F=\bigoplus_{i=1}^{m} F_{i}
$$


Theorem 4.4. Let $K$ be a symmetric cone with irreducible decomposition (4.1). Then the irreducible components $K_{i}$ are symmetric cones. Let $F(x)$ be a self-scaled barrier for $K$. Then there exist self-scaled barrier functions $F_{i}$ for the cones $K_{i}$ such that

$$
F=F_{1} \oplus \cdots \oplus F_{m}
$$

and these functions are unique up to additive constants.

Proof. Recall that (1.7) relates the universal barrier function $U$ to the characteristic function $\varphi_{K}$ of $K$. Since changing the inner product used in the definition of $\varphi_{K}$ changes $U$ only by an additive constant, we may assume that $\langle x, y\rangle=\sum_{i=1}^{m}\left\langle x_{i}, y_{i}\right\rangle_{E_{i}}$ for the purposes of this definition. Here, $\langle\cdot, \cdot\rangle_{E_{i}}$ is an inner product defined on $E_{i}$ chosen so that $U_{i}^{\prime \prime}\left(f_{i}\right)=\mathrm{id}_{E_{i}}$ for some elements $f_{i} \in \operatorname{ri}\left(K_{i}\right)$ where $U_{i}$ denotes the universal barrier function defined on $\operatorname{ri}\left(K_{i}\right)$. Then we have $Q(f)=I$ for $f=\bigoplus_{i=1}^{m} f_{i} \in \operatorname{int}(K)$, in full consistency with our previous notation. Moreover, $K$ is self-dual under $\left\langle\cdot, \cdot \cdot\right.$, since $K^{*}=Q(f) K=K$. Hence, we may choose the vector $e \in \operatorname{int}(K)$ specified in Remark 2.2 as the unique element in $\operatorname{int}(K)$ such that $F^{\prime \prime}(e)=I$ under $\langle\cdot, \cdot\rangle$, see Remark 3.1. The existence of $e$ is guaranteed by Lemma 3.4.

Thus, $U$ can be written as the direct $\operatorname{sum} U(x)=\bigoplus_{i=1}^{m} U_{i}\left(x_{i}\right)$ and $Q(x)$ has a block structure corresponding to the subspaces $E_{i}, Q(x)=\bigoplus_{i=1}^{m} Q_{i}\left(x_{i}\right)$ where $Q_{i}\left(x_{i}\right)=U_{i}^{\prime \prime}\left(x_{i}\right)^{-1}$. Consequently, (3.1) implies that $P(x)$ also has the same block structure

$$
P(x)=P_{1}\left(x_{1}\right) \oplus \cdots \oplus P_{m}\left(x_{m}\right),
$$

where $P_{i}(x)=Q_{i}\left(Q_{i}\left(x_{i}\right)^{1 / 2} e_{i}^{-1}\right) \in \operatorname{Aut}\left(K_{i}\right)$ with $e_{i}^{-1}=\pi_{E_{i}}\left(-F^{\prime}(e)\right), \pi_{E_{i}}$ being the projection defined at the beginning of this section.

So far we know that $P(x)$ has a block structure corresponding to the direct sum $E=\bigoplus_{i=1}^{m} E_{i}$, but it is not a priori clear that $P_{i}\left(x_{i}\right)^{-1}$ is the Hessian of a function defined on $\operatorname{ri}\left(K_{i}\right)$. Let the spaces $\hat{E}_{i}$ be defined as earlier in this section, and let us consider the vector fields $v_{i}: x \mapsto \pi_{E_{i}} F^{\prime}(x)$, defined on int $(K)$ and taking values in $E_{i}$ for all $i, i=1, \ldots, m$. We claim that $v_{i}$ depends only on $x_{i}=\pi_{E_{i}} x$. In fact, for any two vectors $x, y \in \operatorname{int}(K)$ such that $x_{i}=y_{i}$ we have

$$
\begin{aligned}
v_{i}(y) & =\pi_{E_{i}} F^{\prime}(y)=\pi_{E_{i}}\left[F^{\prime}(x)+\int_{0}^{1} F^{\prime \prime}(t y+(1-t) x)[y-x] d t\right] \\
& =v_{i}(x)+\int_{0}^{1} P_{i}\left(\pi_{E_{i}}[t y+(1-t) x]\right)^{-1} \pi_{E_{i}}[y-x] d t=v_{i}(x)+\int_{0}^{1} 0 d t,
\end{aligned}
$$

which shows our claim. Hence, the quotient vector fields

$$
\begin{aligned}
\hat{v}_{i}: \operatorname{int}(K) / \hat{E}_{i} & \rightarrow E_{i}, \\
x \bmod \hat{E}_{i} & \mapsto \pi_{E_{i}} F^{\prime}(x),
\end{aligned}
$$


are well defined and can be identified with vector fields $\hat{v}_{i}$ defined on the cones $\operatorname{ri}\left(K_{i}\right)$. The direct sum of these vector fields amounts to the gradient field

$$
F^{\prime}=\hat{v}_{i} \oplus \cdots \oplus \hat{v}_{m}: \operatorname{int}(K) \rightarrow E .
$$

$F^{\prime}$ being conservative, the $\hat{v}_{i}$ must be conservative too, implying that these are the gradient vector fields of some functions $F_{i}$ defined on $\operatorname{ri}\left(K_{i}\right)$ which are uniquely determined up to additive constants. We may choose these constants so that $F=$ $\bigoplus_{i=1}^{m} F_{i}$. Clearly, we have $F_{i}^{\prime \prime}\left(x_{i}\right)=P_{i}(x)^{-1}$ for any $x \in \operatorname{int}(K)$.

Using (4.3), it is straightforward to check that the $F_{i}$ are self-concordant functions, see [14]. Applying Proposition 1.4(i) and (v) to $F$, using (4.4), and considering variations of $x \in \operatorname{int}(K)$ only in the part $x_{i}=\pi_{E_{i}} x$, we obtain that $\left\langle x_{i},-F_{i}^{\prime}\left(x_{i}\right)\right\rangle=v_{i}$ for some numbers $v_{i}>0$ with $\sum_{i=1}^{m} v_{i}=v$. Moreover, applying Proposition 1.4(ii) to $F$ and using (4.4) we get $F_{i}^{\prime}\left(\tau x_{i}\right)=\tau^{-1} F_{i}^{\prime}\left(x_{i}\right)$ for all $\tau>0$. Hence,

$$
\begin{aligned}
F_{i}\left(\tau x_{i}\right) & =F_{i}\left(x_{i}\right)+\int_{1}^{\tau} \frac{d}{d \xi} F_{i}\left(\xi x_{i}\right) d \xi=F_{i}\left(x_{i}\right)+\int_{1}^{\tau}\left\langle x_{i}, F_{i}^{\prime}\left(\xi x_{i}\right)\right\rangle d \xi \\
& =F_{i}\left(x_{i}\right)-\int_{1}^{\tau} \xi^{-1}\left\langle x_{i},-F_{i}^{\prime}\left(x_{i}\right)\right\rangle d \xi=F_{i}\left(x_{i}\right)-v_{i} \int_{1}^{\tau} \xi^{-1} d \xi \\
& =F_{i}\left(x_{i}\right)-v_{i} \ln \tau .
\end{aligned}
$$

This shows that the functions $F_{i}$ are $v_{i}$-logarithmically homogeneous. It is a wellknown fact that any logarithmically homogeneous self-concordant function is also a barrier function, see, for example, [14] or [18]. It remains to show that the functions $F_{i}$ are self-scaled. As previously noted, condition (2.5) is satisfied, since $P_{i}\left(x_{i}\right) \in \operatorname{Aut}\left(K_{i}\right)$ for all $i$. Finally, condition (2.6) holds for $F_{i}$ because we can apply this condition to $F$, choosing $w=w_{i} \oplus\left(\bigoplus_{j \neq i} e_{i}\right)$ and $x=x_{i} \oplus\left(\bigoplus_{j \neq i} e_{i}\right)$ and using the block structures of $F$ and $F^{\prime \prime}$.

Note that the irreducible components $K_{i}$ of $K$ must be symmetric cones, since the $F_{i}$ are self-scaled barriers defined on $\operatorname{ri}\left(K_{i}\right)$. The symmetry of the $K_{i}$ can also be directly derived from the block structure of $Q(x)=\bigoplus_{i=1}^{m} Q_{i}\left(x_{i}\right)$ and the fact that the set of cone automorphisms $\{Q(x): x \in \operatorname{int}(K)\}$ acts transitively on $\operatorname{int}(K)$.

The decomposition Theorem 4.4 shows that for the purposes of classifying self-scaled barriers we may concentrate our efforts on irreducible cones.

\section{Classification of Self-Scaled Barriers}

In this section, we give a complete classification of self-scaled barrier functions on the symmetric cone $K$.

The definition of a self-scaled barrier function $F$ requires that $F$ changes only by an additive constant under the action of symmetric cone automorphisms $\{P(u): u \in$ 
$\operatorname{int}(K)$ \}, see (2.3). However, it is not a priori known how $F$ behaves under the action of an arbitrary element of $\operatorname{Aut}(K)$. Note that this is in marked contrast to the case where $F$ is the universal barrier function $U$, which is known to change only by an additive constant under the action of any element of the symmetry group of $K$. This explains in a sense the main difficulty one faces when proving the results below.

The next result is a key in resolving this difficulty and is just a slight reformulation of the conjecture raised by Hauser [9], according to which self-scaled barriers on irreducible symmetric cones are isotropic, i.e., invariant under the action of the orthogonal group of $K$. Let us denote by $\operatorname{Aut}(K)_{0}$ the connected component of the identity in $\operatorname{Aut}(K)$.

Theorem 5.1. Let $K$ be a symmetric cone. If $H \in \operatorname{Aut}(K)_{0}$ is orthogonal, then $F(H x)=F(x)$ for all $x \in \operatorname{int}(K)$.

Proof. Koecher [11] proved that if $K$ is a symmetric cone, then $\operatorname{Aut}(K)_{0}$ is generated by $\{Q(u): u \in \mathcal{V}\}$ where $\mathcal{V}$ is a neighborhood of the identity $f$, see [11, Theorem 4.9(b), pp. 88-89]. Koecher's proof exploits the fact that all derivations of the Jordan algebra associated with $U(x)$ are inner. An accessible proof for the case where $K$ is irreducible is given in [1, Lemma VI.1.2, pp. 101-102], based on certain nontrivial results from the theory of Jordan algebras. For a simple independent proof of an equivalent result in the special case of the positive semidefinite symmetric cone, see Hauser [10]. If $H \in \operatorname{Aut}(K)_{0}$ is orthogonal, it follows from Koecher's result that

$$
H=\prod_{1}^{l} Q\left(u_{i}\right)=\prod_{1}^{l} P\left(v_{i}\right),
$$

for some $u_{i}, v_{i} \in \operatorname{int}(K), i=1, \ldots, l$. Here the second equality follows from Lemma 3.4. Therefore, it follows from (2.3) that

$$
F(H x)=F\left(\prod_{1}^{l} P\left(v_{i}\right) x\right)=F(x)+2 \sum_{1}^{l} F\left(v_{i}\right)-2 l F(e) .
$$

Since $H f=f$, setting $x=f$ above yields $\sum_{1}^{l} F\left(v_{i}\right)-2 l F(e)=0$, and this settles the claim of the theorem.

The group $\operatorname{Aut}(K)_{0}$ already acts transitively on $\operatorname{int}(K)$, see [1, p. 5]. Thus, the above result is significant. An immediate consequence of Theorem 5.1 is that, in the case where $K$ is irreducible, $e$ and $f$ are collinear.

Lemma 5.2. If $K$ is irreducible, then $e=\mu f$ for some $\mu>0$.

Proof. Theorem 5.1 implies that

$$
F^{\prime \prime}(H x)=H \circ F^{\prime \prime}(x) \circ H^{*}, \quad \forall H \in O\left(\operatorname{Aut}(K)_{0}\right) .
$$


Since $e$ is characterized by the equation $F^{\prime \prime}(e)=I$, we have in particular $F^{\prime \prime}(H e)=$ $I$, i.e., $H e=e$ for all $H \in O\left(\operatorname{Aut}(K)_{0}\right)$. Moreover, in the case where $K$ is irreducible, the ray generated by $f$ is characterized by the identity

$$
\{\mu f: \mu>0\}=\left\{x \in \operatorname{int}(K): H x=x, \forall H \in O\left(\operatorname{Aut}(K)_{0}\right)\right\},
$$

see, e.g., [1, Prop. III.4.1, p. 51]. The result now follows from this equation.

Nesterov and Todd [15] define two boundary elements $v, w \in \partial K$ to be orthogonal with respect to $z \in \operatorname{int}(K)$ and $F$ if $\langle v, w\rangle_{z}:=\left\langle F^{\prime \prime}(z) v, w\right\rangle=0$. In Theorem 5.1 [15] they prove that in this situation $F$ separates in the directions $v$ and $w$ as follows

$$
F(z+\alpha v+\beta w)=F(z+\alpha v)+F(z+\beta w)-F(z), \quad \forall \alpha, \beta \geq 0 .
$$

Let us now assume that $K$ is irreducible and let $U$ be the universal barrier function for $K$. Let $k$ be the rank of the Jordan algebra associated with $U(x)$, see [1]. Let $x$ be an arbitrary point in $\operatorname{int}(K)$. Then there exists an orthogonal frame $\left\{f_{1}, \ldots, f_{k}\right\}$ such that $f=\sum_{i=1}^{k} f_{i}$ and $x \in \operatorname{int}(C)$, where

$$
C:=\left\{\sum_{i=1}^{k} \alpha_{i} f_{i}: \alpha_{i} \geq 0\right\}
$$

see [1, Theorem III.1.2, pp. 44-45]. Note that $C$ is a direct sum of the half-lines $\left\{\alpha_{i} f_{i}: \alpha_{i} \geq 0\right\}$ and thus a symmetric cone. Moreover, since the $\left\{f_{1}, \ldots, f_{k}\right\}$ are mutually orthogonal with respect to $\langle\cdot, \cdot\rangle=\langle\cdot, \cdot\rangle_{e}$, they are also orthogonal with respect to $f$ and $F$. In fact,

$$
\left\langle f_{i}, f_{j}\right\rangle_{f}=\left\langle F^{\prime \prime}(f) f_{i}, f_{j}\right\rangle \stackrel{\text { Lem } 5.2}{=}\left\langle F^{\prime \prime}(\mu e) f_{i}, f_{j}\right\rangle \stackrel{1.4(\mathrm{iii})}{=} \mu^{-2}\left\langle f_{i}, f_{j}\right\rangle_{e}=0
$$

for all $i \neq j$. We use these properties repeatedly in the proof of the following result.

Lemma 5.3. If $F$ is a self-scaled barrier function defined on the interior of the irreducible symmetric cone $K$, and if $C$ is defined as above, then

$$
F\left(\sum_{i=1}^{k} \alpha_{i} f_{i}\right)=-\frac{v}{k} \sum_{i=1}^{k} \log \alpha_{i}+F(f), \quad \alpha_{i}>0, \quad i=1, \ldots, k
$$

Proof. Let $\sigma$ be any permutation of $\{1, \ldots, k\}$. Theorem IV.2.5 in [1] implies that there exists an orthogonal automorphism $H \in \operatorname{Aut}(K)_{0}$ such that $H f_{i}=f_{\sigma(i)}$, $i=1, \ldots, k$. Using Theorem 5.1, we then obtain

$$
F\left(\sum_{i=1}^{k} \alpha_{i} f_{\sigma(i)}\right)=F\left(\sum_{i=1}^{k} \alpha_{i} f_{i}\right), \quad \forall \alpha_{i}>0, \quad i=1, \ldots, k .
$$


Define $g\left(\alpha_{1}, \ldots, \alpha_{k}\right):=F\left(\sum_{i=1}^{k} \alpha_{i} f_{i}\right)$. We have just shown that $g$ is a symmetric function. Consider a point $f+\sum_{i=1}^{k} \beta_{i} f_{i}=\sum_{i=1}^{k} \alpha_{i} f_{i} \in \mathrm{ri}(C)$, with arbitrary $\beta_{i} \geq 0$ and $\alpha_{i}:=1+\beta_{i}$. Applying the separation property from (5.1) repeatedly, we obtain

$$
F\left(f+\sum_{i=1}^{k} \beta_{i} f_{i}\right)-F(f)=\sum_{i=1}^{k}\left(F\left(f+\beta_{i} f_{i}\right)-F(f)\right) .
$$

Using the symmetry of $g$, the above equation translates into

$$
\begin{aligned}
g\left(\alpha_{1}, \ldots, \alpha_{k}\right)-F(f)= & \sum_{i=1}^{k}\left(g\left(\alpha_{i}, 1, \ldots, 1\right)-F(f)\right), \\
& \forall \alpha_{i} \geq 1, \quad i=1, \ldots, k .
\end{aligned}
$$

If $\alpha_{1}=\cdots=\alpha_{k}=\alpha$ above, we have

$$
g(\alpha, \ldots, \alpha)-F(f)=F(\alpha f)-F(f)=F(f)-v \log \alpha-F(f)=-v \log \alpha .
$$

This yields $g(\alpha, 1, \ldots, 1)-F(f)=-(\nu / k) \log \alpha$ for all $\alpha \geq 1$. Consequently, we have

$$
g\left(\alpha_{1}, \ldots, \alpha_{k}\right)-F(f)=-\frac{v}{k} \sum_{i=1}^{k} \log \alpha_{i}, \quad \forall \alpha_{i} \geq 1, \quad i=1, \ldots, k .
$$

Now, if $\alpha_{i}>0, i=1, \ldots, k$, are arbitrary, choose $t>0$ such that $\hat{\alpha}_{i}=\alpha_{i} / t \geq 1$ for all $i$. Since $F$ is logarithmically homogeneous, we have $g\left(\alpha_{1}, \ldots, \alpha_{k}\right)=$ $g\left(\hat{\alpha}_{1}, \ldots, \hat{\alpha}_{k}\right)-v \log t$. A simple calculation now shows that (5.2) holds true for all $\alpha_{i}>0$.

The following theorem classifies self-scaled barrier functions for irreducible symmetric cones.

Theorem 5.4. Let $K$ be an irreducible symmetric cone, and let $F$ be a self-scaled barrier function defined on $\operatorname{int}(K)$. Then there exist constants $\alpha>0$ and $\beta$ such that

$$
F(x)=\alpha U(x)+\beta,
$$

where $U(x)$ is the universal barrier function on int $K$.

Proof. Lemma 5.3 describes the restriction of $F$ on ri $(C)$. Since the universal barrier function is also self-scaled, the same considerations apply to $U(x)$. Thus, the functions $F$ and $U$ are homothetic transformations of each other on $\operatorname{ri}(C)$, that is, there exist $\alpha>0, \beta$ such that

$$
F(x)=\alpha U(x)+\beta .
$$


Let $y \in \operatorname{int}(K)$ be an arbitrary point with the spectral decomposition $y=$ $\sum_{i=1}^{k} \lambda_{i} y^{(i)}$. Corollary IV.2.7 in [1] implies that there exists $H \in O\left(\operatorname{Aut}(K)_{0}\right)$ such that $H f_{i}=y^{(i)}, i=1, \ldots, k$. We have $y=H x$ where $x=\sum_{i=1}^{k} \lambda_{i} f_{i} \in \operatorname{ri}(C)$. Theorem 5.1 yields $F(y)=F(x)$ and $U(y)=U(x)$, and hence the identity (5.3) extends to all of $\operatorname{int}(K)$.

We are now ready to give the final classification theorem for self-scaled barrier functions on arbitrary symmetric cones. This theorem shows that all self-scaled barrier functions are related to the standard logarithmic or the universal barrier via homothetic transformations.

Theorem 5.5. Let $F$ be a self-scaled barrier function for a symmetric cone $K$ with irreducible decomposition (4.1). Then there exist constants $c_{0}$ and $c_{1} \geq$ $1, \ldots, c_{m} \geq 1$ such that

$$
F=c_{0}-\bigoplus_{i=1}^{m} c_{i} \ln \operatorname{det}_{K_{i}}
$$

see (4.2). Conversely, any function of this form is a self-scaled barrier for $K$.

Here $\operatorname{det}_{K_{i}} x_{i}$ denotes the determinant of $x_{i} \in \operatorname{ri}\left(K_{i}\right)$ in the Jordan algebraic sense, see [1, Chap. 2].

Proof. Theorems 4.4 and 5.4 imply that there exist constants $d_{0}$ and $d_{1}>$ $0, \ldots, d_{m}>0$ such that

$$
F(x)=d_{0}+d_{1} u_{1}\left(x_{1}\right)+\cdots+d_{m} u_{m}\left(x_{m}\right),
$$

where $u_{i}\left(x_{i}\right)=\ln \varphi_{K_{i}}\left(x_{i}\right)$. It is known that $u_{i}\left(x_{i}\right)=$ const $-\left(n_{i} / r_{i}\right) \ln \operatorname{det}_{K_{i}} x_{i}$, where $r_{i}$ is the rank of the Jordan algebra associated with $u_{i}(x)$, and $n_{i}$ is the dimension of the cone $K_{i}$, see [1, Prop. III.4.3, p. 53]. Finally, Theorem 4.1 in [7] implies that the function $-\alpha \ln \operatorname{det}_{K_{i}} x_{i}$ is self-concordant if and only if $\alpha \geq 1$.

Remark 5.6. In interior-point methods based on barrier functions, the complexity parameter $v$ of the barrier plays a crucial role in the bound on the number of iterations necessary to approximate an optimal solution to a given level of accuracy. The quest for a barrier with minimal possible complexity parameter is therefore an important issue. If $K$ is an irreducible symmetric cone, then det $x$ is a homogeneous polynomial with degree equal to the rank of $K$, see [1, Sect. II.2]. Thus, $F(x)=-\ln \operatorname{det} x$ is a self-scaled barrier with $v$ equal to the rank of $K$. It then follows from Theorem 4.1 in Güler and Tunçel [7] that this barrier has optimal parameter $v$. Therefore, the optimal self-scaled barrier in Theorem 5.5 has $c_{i}=1$, $i=1, \ldots, m$. 


\section{Acknowledgments}

Our thanks go to the three anonymous referees and the associate editor who carefully read our manuscript several times and suggested numerous important improvements.

Raphael Hauser conducted his research on this paper partially while writing his $\mathrm{PhD}$ at the School of Operations Research and Industrial Engineering of Cornell University, and partially as a post-doctoral fellow at the University of Cambridge. He wishes to thank Mike Todd, Jim Renegar, and Arieh Iserles for numerous valuable discussions and for their continuous support. This research was supported by a "bourse pour chercheur débutant" from the Swiss National Science Foundation and the Swiss Academy of Technical Sciences, and by the Engineering and Physical Sciences Research Council of the United Kingdom, EPSRC grant GR/M30975.

The research of Osman Güler on this paper was conducted at the Industrial Engineering Department at Bilkent University, Ankara, Turkey, while on a sabbatical leave from the Department of Mathematics and Statistics, University of Maryland, Baltimore County, Baltimore, Maryland. This author thanks the Industrial Engineering Department at Bilkent University for providing him a congenial atmosphere and excellent working conditions. This research was partially supported by the National Science Foundation under grant DMS-0075722.

\section{References}

[1] J. Faraut and A. Korányi, Analysis on Symmetric Cones, Oxford University Press, Oxford, UK, 1994.

[2] L. Faybusovich, Jordan algebras, symmetric cones and interior-point methods, Technical Report, Department of Mathematics, University of Notre Dame, IN, October 1995.

[3] P. M. Gruber, Zur Charakterisierung konvexer Körper. Über einen Satz von Rogers und Shepard II, Math. Ann. 184 (1970), 79-105.

[4] O. Güler, Private communication to Y. E. Nesterov and M. J. Todd, April 1994.

[5] O. Güler, Barrier functions in interior point methods, Math. Oper. Res. 21 (1996), 860-885.

[6] O. Güler, Interior-point methods on symmetric and homogeneous cones, Presentation, 17th International Symposium on Mathematical Programming, Atlanta, Georgia, 7-11 August, 2000.

[7] O. Güler and L. Tunçel, Characterization of the barrier parameter of homogeneous convex cones, Math. Programming (Series A) 81 (1998), 55-76.

[8] R. A. Hauser, On Search Directions for Self-Scaled Conic Programming, Ph.D. thesis, School of Operations Research and Industrial Engineering, Cornell University, January 2000.

[9] R. A. Hauser, Self-Scaled Barrier Functions: Decomposition and Classification, Numerical Analysis Report DAMTP 1999/NA13, Department of Applied Mathematics and Theoretical Physics, Silver Street, Cambridge CB3 9EW, England, October 1999.

[10] R. A. Hauser, Self-Scaled Barriers for Semidefinite Programming, Numerical Analysis Report DAMTP 2000/NA02, Department of Applied Mathematics and Theoretical Physics, Silver Street, Cambridge CB3 9EW England, March 2000.

[11] M. Koecher, The Minnesota Notes on Jordan Algebras and their Applications, Lecture Notes in Mathematics, Vol. 1710, Springer-Verlag, New York, 1999.

[12] Y. Lim, Private communication to L. Faybusovich, Spring 2000.

[13] K. McCrimmon, Norms and noncommutative Jordan algebras, Pacific J. Math. 15 (1965), 925956. 
[14] Y. E. Nesterov and A. S. Nemirovskii, Interior-Point Polynomial Algorithms in Convex Programming, SIAM, Philadelphia, 1994.

[15] Y. E. Nesterov and M. J. Todd, Self-scaled barriers and interior-point methods for convex programming, Math. Oper. Res. 22 (1997), 1-42.

[16] Y. E. Nesterov and M. J. Todd, Primal-dual interior-point methods for self-scaled cones, SIAM J. Optim. 8 (1998), 324-364.

[17] H. P. Petersson, Max Koecher's work on Jordan algebras, in Jordan Algebras (W. Kaup, K. McCrimmon, and H. L. Petersen, eds.), de Gruyter, Berlin, 1995, pp. 187-195.

[18] J. Renegar, A Mathematical View of Interior-Point Methods in Convex Optimization, SIAM, Philadelphia, 2001.

[19] O. S. Rothaus, Domains of positivity, Abh. Math. Sem. Univ. Hamburg 24 (1960), 189-235.

[20] S. H. Schmieta, Complete classification of self-scaled barrier functions, Technical Report, Department of IEOR, Columbia University, New York, NY 10027, July 2000.

[21] È. B. Vinberg, The theory of homogeneous convex cones, Trans. Moscow Math. Soc. 12 (1963), 340-403. 